\title{
Multihadron production dynamics exploring the energy balance in hadronic and nuclear collisions
}

\author{
Edward K. G. Sarkisyan, ${ }^{1,2, *}$ Aditya Nath Mishra, ${ }^{3, \dagger}$ Raghunath Sahoo, ${ }^{3, \ddagger}$ and Alexander S. Sakharov ${ }^{1,4,5,8}$ \\ ${ }^{1}$ Experimental Physics Department, CERN, 1211 Geneva 23, Switzerland \\ ${ }^{2}$ Department of Physics, The University of Texas at Arlington, Arlington, Texas 76019, USA \\ ${ }^{3}$ Discipline of Physics, School of Basic Sciences, Indian Institute of Technology Indore, \\ Indore 452020, India \\ ${ }^{4}$ Department of Physics, New York University, New York, New York 10003, USA \\ ${ }^{5}$ Physics Department, Manhattan College, Riverdale, New York 10471, USA \\ (Received 27 January 2016; published 30 March 2016; corrected 11 April 2016)
}

\begin{abstract}
The relation of multihadron production in nucleus-nucleus and (anti)proton-proton collisions is studied by exploring the collision-energy and centrality dependencies of the charged particle mean multiplicity in the measurements to date. The study is performed in the framework of the recently proposed effectiveenergy approach which, under the proper scaling of the collision energy, combines the constituent quark picture with Landau relativistic hydrodynamics counting for the centrality-defined effective energy of participants. Within this approach, the multiplicity energy dependence and the pseudorapidity spectra from the most central nuclear collisions are well reproduced. The study of the multiplicity centrality dependence reveals a new scaling between the measured pseudorapidity spectra and the calculations. By means of this scaling, referred to as energy-balanced limiting fragmentation scaling, one reproduces the pseudorapidity spectra for all centralities. The scaling elucidates some differences in the multiplicity and midrapidity density centrality dependence obtained at RHIC and LHC. These findings reveal an inherent similarity in the multiplicity energy dependence from the most central collisions and centrality data. Predictions are made for the mean multiplicities to be measured in proton-proton and heavy-ion collisions at the LHC.
\end{abstract}

DOI: 10.1103/PhysRevD.93.054046

\section{INTRODUCTION}

Study of global observables of multiparticle production and their universality in different types of high-energy collisions is of a crucial importance for understanding the underlying dynamics of strong interactions. Recently, the universality of multiparticle production in nucleus-nucleus and hadron-hadron collisions has been reported exploiting concept of centrality-defined effective energy [1] employed for the data interpreted in terms of the approach of the dissipating energy of quark participants [2,3]. This approach combines the constituent quark picture together with Landau relativistic hydrodynamics and interrelates multihadron production in different types of collisions. Within this picture, one can successfully explain $[2,3]$ the scaling between the charged particle mean multiplicity in $e^{+} e^{-}$and $p p / \bar{p} p$ collisions [4] and the universality of both the multiplicity and the midrapidity pseudorapidity density

*sedward@cern.ch

†Aditya.Nath.Mishra@cern.ch

\#Raghunath.Sahoo@cern.ch

§Alexandre.Sakharov@cern.ch

Published by the American Physical Society under the terms of the Creative Commons Attribution 3.0 License. Further distribution of this work must maintain attribution to the author(s) and the published article's title, journal citation, and DOI. measured in the most central nuclear collisions and in $e^{+} e^{-}$ annihilation [5]. ${ }^{1}$ The universality of the multihadron production irrespective of the collision species, an intrinsic feature of the dissipating energy approach, is widely discussed nowadays [6-8].

In this paper, in the framework of the approach of the dissipating effective energy of constituent quark participants, or, for brevity, the participant dissipating energy (PDE) approach, we extend the previous studies of the charged particle mean multiplicity $[2,3]$ to LHC energies. We show that the multiplicity energy dependence of headon collisions is well described within the proposed approach. In addition, here we study the dependence of the multiplicity on the number of (nucleon) participants at RHIC and LHC. We introduce a new scaling, referred to as the energy-balanced limiting fragmentation scaling, which allows us to describe the pseudorapidity density spectra independently of the centrality of collisions. Using this scaling, a complementarity between the multiplicities measured in head-on nuclear collisions and obtained from the centrality data is found. The study clarifies some differences of the centrality dependence of multiplicities

\footnotetext{
${ }^{1}$ Elsewhere in this paper, the multiplicity is defined as being averaged over events in what is referred to as the "mean" multiplicity. No other averaging, e.g. over centralities in nuclear data, is considered.
} 
measured at RHIC and LHC. Finally, predictions are made for the charged particle mean multiplicities in $p p$ and heavy-ion collisions at the LHC.

\section{THE PARTICIPANT DISSIPATING ENERGY APPROACH}

In this section, we briefly describe the PDE approach, as it is proposed in [1-3]. This approach quantifies the process of particle production in terms of the amount of energy deposited by interacting constituent quark participants inside the small Lorentz-contracted volume formed at the early stage of a collision. The whole process of a collision is then represented as the expansion of an initial state and the subsequent breakup into particles. This approach resembles the Landau phenomenological hydrodynamic approach of multiparticle production in relativistic particle interactions [9], which was found to be in a good agreement with the multiplicity data in particle and nuclear collisions in the wide energy range [10]. In the picture considered here, the Landau hydrodynamics is employed in the framework of constituent (or dressed) quarks, in accordance with the additive quark model [11-14]; for a recent comprehensive review on soft hadron interactions in the additive quark model, see [15]. This means the secondary particle production is basically driven by the amount of the initial effective energy deposited by constituent quarks into the Lorentz-contracted region. In $p p / \bar{p} p$ collisions, a single constituent quark from each nucleon is considered to take part in a collision, and the remaining quarks are treated as spectators. The spectator quarks do not participate in the secondary particle production, but they result in a formation of leading particles and carry away a significant part of the collision energy. Thus, the effective energy for the production of secondary particles is the energy of interaction of a single quark pair, i.e. $1 / 3$ of the entire nucleon energy. On the contrary, in the head-on heavy-ion collisions, the participating nucleons are considered colliding with all three constituent quarks from each nucleon. This makes the whole energy of the colliding nucleons (participants) available for the secondary particle production. Within this picture, one expects the results for bulk observables from head-on heavy-ion collisions at the c.m. energy per nucleon, $\sqrt{s_{N N}}$, to be similar to those from the $p p / \bar{p} p$ collisions but corresponding to a 3 times larger c.m. energy, i.e. at $\sqrt{s_{p p}} \simeq 3 \sqrt{s_{N N}}$. Such a universality is found to correctly predict [2] the value of the midrapidity density in $p p$ interactions measured at the TeV LHC energies [16]. In addition, the multiplicity measurements in $p p / \bar{p} p$ interactions up to $\mathrm{TeV}$ energies are shown to be well reproduced by $e^{+} e^{-}$data as soon as the inelasticity is set to $\approx 0.35$ [7], i.e. effectively $1 / 3$ of the hadronic interaction energy. This is in agreement with the dissipation energy picture where the structureless colliding leptons are considered to deposit their total energy into the Lorentzcontracted volume, similarly to nucleons in head-on nuclear collisions [2]. For a recent discussion on the universality of hadroproduction up to LHC energies, see [8].

Combining the above-discussed ingredients of the constituent quark picture and Landau hydrodynamics, one obtains the relationship between charged particle rapidity density per participant pair, $\rho(\eta)=\left(2 / N_{\text {part }}\right) d N_{\text {ch }} / d \eta$ at midrapidity $(\eta \approx 0)$, in heavy-ion collisions and in $p p / \bar{p} p$ collisions:

$$
\frac{\rho(0)}{\rho_{p p}(0)}=\frac{2 N_{\mathrm{ch}}}{N_{\mathrm{part}} N_{\mathrm{ch}}^{p p}} \sqrt{\frac{L_{p p}}{L_{N N}}}, \quad \sqrt{s_{p p}}=3 \sqrt{s_{N N}} .
$$

In Eq. (1), the relation of the pseudorapidity density and the mean multiplicity is applied in its Gaussian form as obtained in Landau hydrodynamics. The factor $L$ is defined as $L=\ln (\sqrt{s} / 2 m)$. According to the approach considered, $m$ is the proton mass, $m_{p}$, in nucleus-nucleus collisions and the constituent quark mass in $p p / \bar{p} p$ collisions set to $\frac{1}{3} m_{p}$. $N_{\mathrm{ch}}$ and $N_{\mathrm{ch}}^{p p}$ are the mean multiplicities in nucleus-nucleus and nucleon-nucleon collisions, respectively, and $N_{\text {part }}$ is the number of participants.

Solving Eq. (1) for the multiplicity $N_{\mathrm{ch}}$ at a given rapidity density $\rho(0)$ at $\sqrt{s_{N N}}$, and for the rapidity density $\rho_{p p}(0)$ and the multiplicity $N_{\mathrm{ch}}^{p p}$ at $3 \sqrt{s_{N N}}$, one finds

$$
\begin{aligned}
\frac{2 N_{\mathrm{ch}}}{N_{\mathrm{part}}} & =N_{\mathrm{ch}}^{p p} \frac{\rho(0)}{\rho_{p p}(0)} \sqrt{1-\frac{2 \ln 3}{\ln \left(4.5 \sqrt{s_{N N}} / m_{p}\right)}}, \\
\sqrt{s_{N N}} & =\sqrt{s_{p p}} / 3 .
\end{aligned}
$$

Further development, as outlined below, treats this dependence in terms of centrality [1]. The centrality is regarded as the degree of the overlap of the volumes of the two colliding nuclei, characterized by the impact parameter. The most central collisions correspond, therefore, to the lowest centrality while the larger centrality to the more peripheral collisions. The centrality is closely related to the number of nucleon participants determined using Monte Carlo Glauber calculations. Hence, the largest number of participants contribute to the most central heavy-ion collisions. The centrality is thus related to the amount of energy released in the collisions, i.e. to the effective energy, $\varepsilon_{N N}$. The latter, in the framework of the proposed approach, can be defined as a fraction of the c.m. energy available in a collision according to the centrality, $\alpha$ :

$$
\varepsilon_{N N}=\sqrt{s_{N N}}(1-\alpha) .
$$

Conventionally, the data are divided into centrality intervals, so that $\alpha$ is the average centrality per centrality interval, e.g. $\alpha=0.25$ for the centrality interval of $20 \%-$ $30 \%$ centrality. 
Then, for the effective c.m. energy $\varepsilon_{N N}$, Eq. (2) reads

$$
\begin{aligned}
\frac{2 N_{\mathrm{ch}}}{N_{\mathrm{part}}} & =N_{\mathrm{ch}}^{p p} \frac{\rho(0)}{\rho_{p p}(0)} \sqrt{1-\frac{2 \ln 3}{\ln \left(4.5 \varepsilon_{N N} / m_{p}\right)}}, \\
\varepsilon_{N N} & =\sqrt{s_{p p}} / 3
\end{aligned}
$$

where $\rho(0)$ is the midrapidity density in central nucleusnucleus collisions measured at $\sqrt{s_{N N}}=\varepsilon_{N N}$.

In fact, each of the scalings described by Eqs. (2) and (3) regulates a particular physics ingredient used in the modelling of the PDE approach. The scaling introduced by Eq. (2) embeds the constituent quark model, which leads to establishing a similarity between hadronic and nuclear collisions. The scaling driven by Eq. (3) addresses the energy budget effectively retained in the most central collisions while considering the global variables from noncentral collisions.

\section{MULTIPLICITY C.M. ENERGY DEPENDENCE IN CENTRAL NUCLEAR AND $p p / \bar{p} p$ COLLISIONS}

Figure 1 shows the c.m. energy dependence of the multiplicity measured in head-on nucleus-nucleus collisions (solid symbols) in the energy range of $\sqrt{s_{N N}}=$ $2 \mathrm{GeV}$ to $2.76 \mathrm{TeV}$. Given the fact that the measurements support the second-order logarithmic dependence on $\sqrt{s_{N N}}$ up to the top RHIC energy $[2,19]$ while the power-law dependence is obtained for the LHC data [17], we fit the head-on data by the "hybrid" fit function:

$$
\begin{aligned}
\frac{2 N_{\mathrm{ch}}}{N_{\text {part }}}= & (-0.577 \pm 0.177)+(0.394 \pm 0.094) \ln \left(s_{N N} / s_{0}\right) \\
& +(0.213 \pm 0.014) \ln ^{2}\left(s_{N N} / s_{0}\right) \\
& +(0.005 \pm 0.009)\left(s_{N N} / s_{0}\right)^{(0.55 \pm 0.11)}
\end{aligned}
$$

Here, $s_{0}=1 \mathrm{GeV}^{2}$. In the following, the factor $s_{0}$ is taken the same in all fit functions and omitted for brevity. This fit is shown in Fig. 1 by the solid line. Note that from the theoretical description point of view, the logarithmic dependence is considered to characterize the fragmentation source(s) while the power-law behavior is believed to come from the gluon-gluon interactions [32]; for a review, see [33].

We also fit the head-on collision multiplicities with the power-law function. The power-law dependence of the multiplicity is expected in different theoretical approaches [34-36], and the data from nuclear and $p p / \bar{p} p$ collisions seem to follow this type of behavior at higher energies $[7,17]$. The power-law fit gives

$\frac{2 N_{\mathrm{ch}}}{N_{\text {part }}}=(-6.72 \pm 1.44)+(5.42 \pm 1.11) s_{N N}^{(0.18 \pm 0.02)}$,

and is shown by the dashed-dotted line in Fig. 1.
Recently, it was shown [37] that the multiplicity of the gluon-gluon interactions are better described within a nonequilibrium statistical relativistic diffusion model using $\log ^{3}\left(s_{N N}\right)$ dependence. Using this behavior, we fit the c.m. energy dependence by the corresponding fit function,

$$
\begin{aligned}
\frac{2 N_{\mathrm{ch}}}{N_{\mathrm{part}}}= & (0.72 \pm 1.85)+(0.75 \pm 0.39) \ln \left(s_{N N}\right) \\
& +(0.019 \pm 0.002) \ln ^{3}\left(s_{N N}\right) .
\end{aligned}
$$

Here, the linear-log term reflects the multiplicity from the fragmentation sources, as noticed above. The fit is shown by the pink solid line in Fig. 1. The fit is made starting the lowest NA49 energy in order to match the LHC multiplicity. One can see that the fit seems to be indistinguishable from the power-law function for the entire fit range, and is slightly below the power-law behavior above the current LHC data. Some enhancement in the low-energy range is expected due to no gluonic source considered to be present at these energies.

In addition to these fits, we show the $\log ^{2}\left(s_{N N}\right)$-fit $[2,3]$ up to the top RHIC energy (thin dashed line). One can see that the power-law fit well describes the data and is almost indistinguishable from the hybrid fit up to the LHC data. Some minor deviation between the two fits can be seen in the range from the top RHIC energy to the LHC energy. Meantime, the second-order log polynomial lies below the data for $\sqrt{s_{N N}}>200 \mathrm{GeV}$. This observation supports a possible transition to a new regime in heavy-ion collisions at $\sqrt{s_{N N}}$ of about $1 \mathrm{TeV}$, as indicated earlier in the studies of pseudorapidity particle and transverse energy densities at midrapidity [1].

Addressing now Eq. (2), we calculate the mean multiplicity $N_{\mathrm{ch}} /\left(N_{\text {part }} / 2\right)$ for nucleus-nucleus interactions using the $p p / \bar{p} p$ measurements. The calculated values are shown in Fig. 1 by large open symbols. The rapidity density $\rho_{p p}(0)$ and the multiplicity $N_{\mathrm{ch}}^{p p}$ are taken from the existing data [8] or, where not available, calculated using the corresponding experimental $\sqrt{s_{p p}}$ fits $^{2}$ at $\sqrt{s_{p p}}=$ $3 \sqrt{s_{N N}}$, in accordance with the approach considered here. The $\rho(0)$ values are as well taken from the measurements in central heavy-ion collisions wherever available, while for the nonexisting data the experimental $\mathrm{fit}^{3}$ is used.

One can see that the calculated $N_{\mathrm{ch}} /\left(0.5 N_{\text {part }}\right)$ values follow the measurements from nucleus-nucleus collisions

\footnotetext{
${ }^{2}$ The powewr-law fit, Eq. (9), is used for $N_{\mathrm{ch}}^{p p}$, while $\rho_{p p}(0)$ is calculated using the linear-log fit $\rho_{p p}(0)=-0.308+$ $0.276 \ln \left(s_{p p}\right)$ [7] and the power-law fit by CMS [38], $\rho_{p p}(0)=$ $-0.402+s_{p p}^{0.101}$, at $\sqrt{s_{p p}} \leq 53 \mathrm{GeV}$ and at $\sqrt{s_{p p}}>53 \mathrm{GeV}$, respectively.

${ }^{3}$ The linear-log fit $\rho(0)=-0.33+0.38 \ln \left(s_{N N}\right)$ [2,19] is applied at $\sqrt{s_{N N}} \leq 63 \mathrm{GeV}$, and the power-law fit $\rho(0)=$ $0.73 s_{N N}^{0.155}$ is applied above $63 \mathrm{GeV}$ as recently reported by ALICE using the measurements up to $\sqrt{s_{N N}}=5.02 \mathrm{TeV}$ [39].
} 


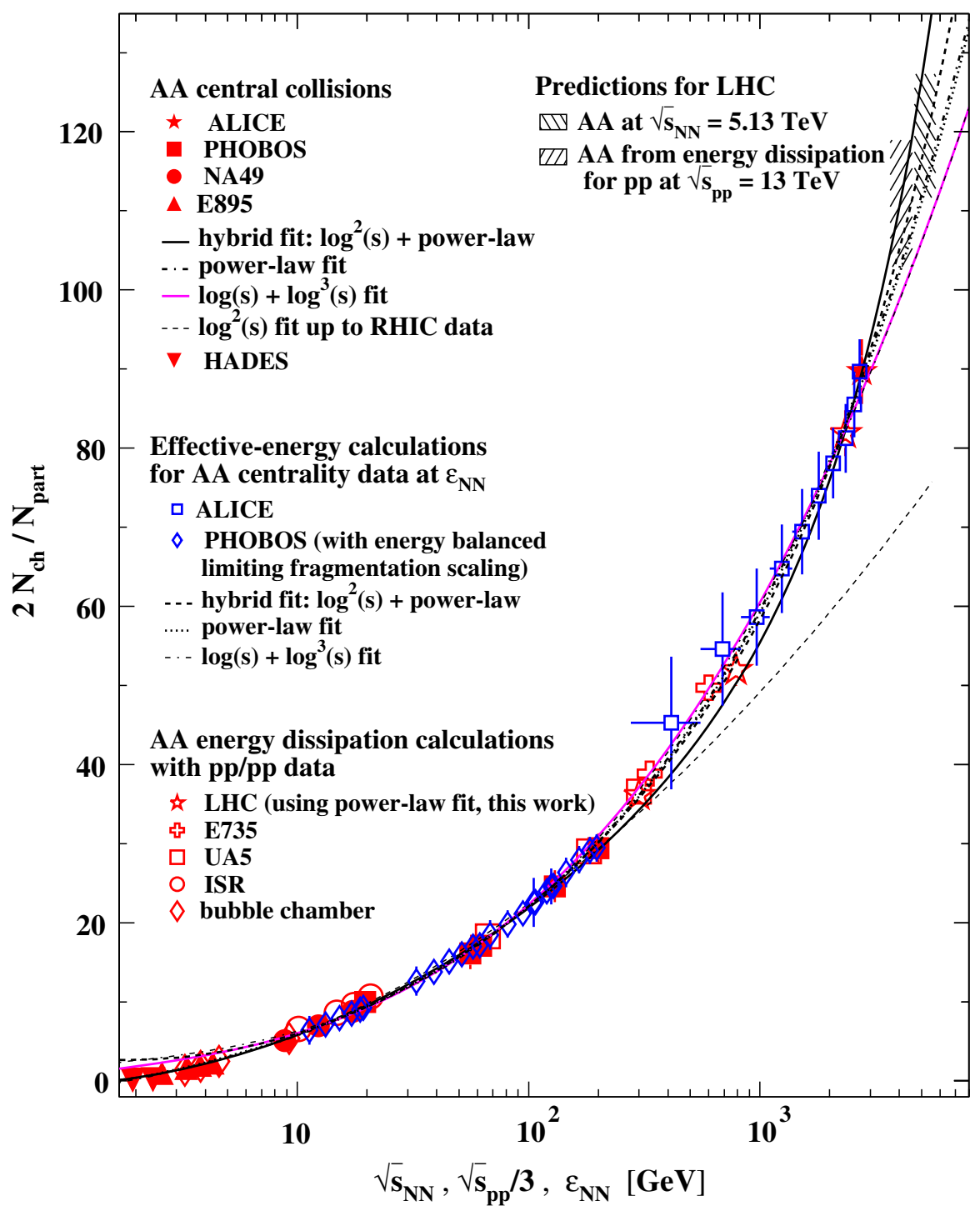

FIG. 1. The energy dependence of the charged particle mean multiplicity per participant pair. The large solid symbols show the measurements from the most central nucleus-nucleus (AA) collisions given as a function of the nucleon-nucleon c.m. energy, $\sqrt{s_{N N}}$. The calculations by Eq. (2) based on $p p / \bar{p} p$ data at the c.m. energy $\sqrt{s_{p p}}=3 \sqrt{s_{N N}}$ are shown vs $\sqrt{s_{p p}} / 3$ by large open symbols. The small open symbols show the AA data at different centralities as a function of the effective energy $\varepsilon_{N N}$ [Eq. (3)]. The RHIC centrality data are shown after removing the energy-balanced limiting fragmentation scaling ingredient, while this ingredient is not taken into account for the LHC centrality data (see text). The multiplicity data of the most-central AA collisions are measured by the ALICE experiment at LHC [17], by the PHOBOS experiment at RHIC [18,19], by the NA49 experiment at CERN SPS [20] and by the E895 experiment at AGS [21] (for the latter see also [18]). The low-energy HADES measurements at GSI are taken from [22]. The centrality data are taken from the measurements by the PHOBOS experiment at RHIC [19] and by the ALICE experiment at the LHC [17,23]. The values obtained from Eq. (2) for the AA mean multiplicity are based on: nonsingle diffractive $\bar{p} p$ collisions at FNAL by the E735 experiment [7,24], at CERN by the UA5 experiment at $\sqrt{s_{p p}}=546 \mathrm{GeV}$ [25] and $\sqrt{s_{p p}}=200$ and $900 \mathrm{GeV}$ [26]; pp collisions from CERN-ISR [27], and from the inelastic data from the bubble chamber experiments [28-30], the latter having been compiled and analyzed in [31]. The LHC multiplicities in $p p$ interactions are calculated using the hybrid fit obtained here, Eq. (9). The solid and the dashed-dotted show, correspondingly, the hybrid fit, $-0.577+0.394 \ln \left(s_{N N}\right)+0.213 \ln ^{2}\left(s_{N N}\right)+0.005 s_{N N}^{0.551}$, and the power-law fit, $-6.72+5.42 s_{N N}^{0.18}$, to the most central AA data. The thin dashed line shows the second-order $\log$ fit $-0.35+0.24 \ln \left(s_{N N}\right)+$ $0.24 \ln ^{2}\left(s_{N N}\right)$ to the most central AA data up to the top RHIC energy [2,3]. The dashed and the dotted lines show, correspondingly, the hybrid fit, $3.04-1.4 \ln \left(\varepsilon_{N N}\right)+1.12 \ln ^{2}\left(\varepsilon_{N N}\right)+0.032 \varepsilon_{N N}^{0.848}$, and the power-law fit, $-6.62+5.43 \varepsilon_{N N}^{0.362}$, to the centrality AA data. The pink solid line and the thin dashed-dotted line show the fits $0.72+0.75 \ln \left(s_{N N}\right)+0.019 \ln ^{3}\left(s_{N N}\right)$ and $1.7+2.36 \ln \left(\varepsilon_{N N}\right)+$ $0.152 \ln ^{3}\left(\varepsilon_{N N}\right)$ to the most central collision and centrality AA data, respectively (see text). The right-inclined hatched area shows the prediction for heavy-ion collisions at $\sqrt{s_{N N}}=5.13 \mathrm{TeV}$ and the left-inclined hatched area gives the prediction expected from $p p$ collisions at $\sqrt{s_{p p}}=13 \mathrm{TeV}$. 


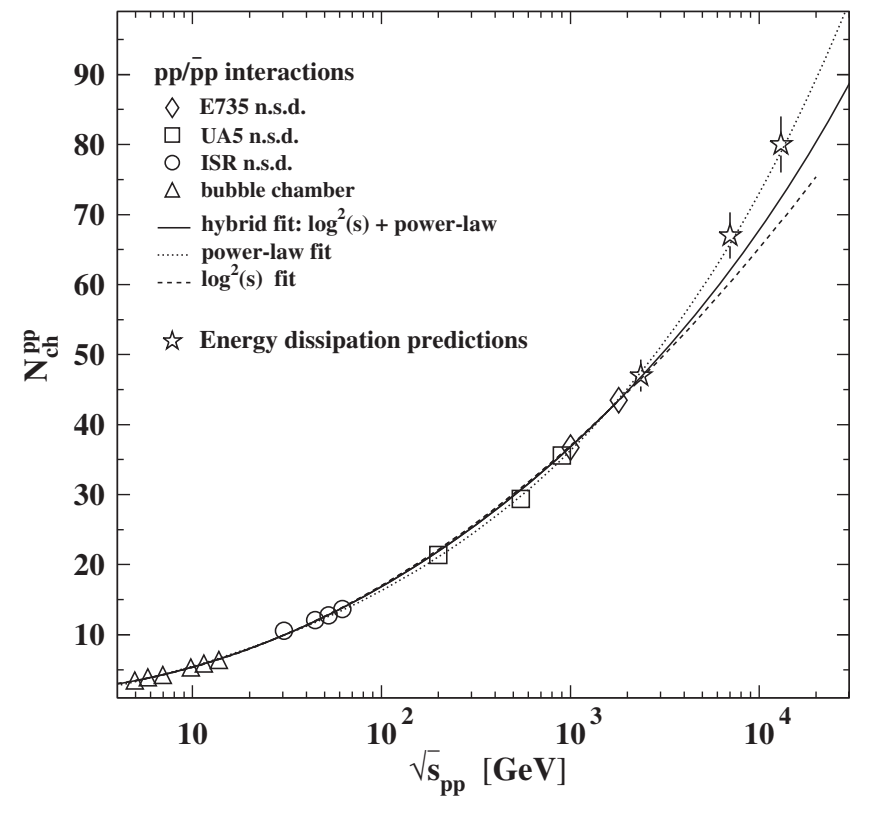

FIG. 2. The c.m. energy dependence of the charged particle mean multiplicity in $p p / \bar{p} p$ collisions. The measurements are taken from: nonsingle diffractive $\bar{p} p$ collisions at FNAL by the E735 experiment $[7,24]$, at CERN by the UA5 experiment at $\sqrt{s_{p p}}=$ $546 \mathrm{GeV}$ [25] and $\sqrt{s_{p p}}=200$ and $900 \mathrm{GeV}$ [26]; $p p$ collisions from CERN-ISR [27], and from the inelastic data from the bubble chamber experiments $[28-31,44]$. The solid line shows the hybrid fit, $1.60-0.03 \ln \left(s_{p p}\right)+0.18 \ln ^{2}\left(s_{p p}\right)+0.03 s_{p p}^{0.29}$, the dotted line shows the power-law fit, $-7.36+6.97 s_{p p}^{0.133}$, and the dashed line shows the second-order $\log$ fit, $3.18-0.57 \ln \left(s_{p p}\right)+$ $0.216 \ln ^{2}\left(s_{p p}\right)$. The open stars show the predictions by the PDE approach with the error bars of $5 \%$ uncertainty.

at $\sqrt{s_{N N}}$ from a few GeV up to the TeV LHC energy. The observed agreement between the heavy-ion measurements of $N_{\mathrm{ch}} /\left(N_{\mathrm{part}} / 2\right)$ and the values obtained from the $p p$ based calculations points to the universality of the multiparticle production process in different types of collisions.

Solving Eq. (2) for the mean multiplicity $N_{\mathrm{ch}}^{p p}$ in $p p$ collisions, we estimate its values for $\sqrt{s_{p p}}>2 \mathrm{TeV}$ to be about 47 at $\sqrt{s_{p p}}=2.36 \mathrm{TeV}, 67$ at $7 \mathrm{TeV}$, and 79 at $13 \mathrm{TeV}$ with 5\% uncertainties. Here for the calculations, one uses the fit to the heavy-ion midrapidity density data $\rho(0)$, as described above, and the fit by ALICE to the headon heavy-ion data on the mean multiplicity [17] [similar to the results for the fits of Eqs. (5) and (6)], along with the LHC measurements [40-43] of the pseudorapidity density $\rho_{p p}(0)$. The calculated values of $N_{\mathrm{ch}}^{p p}$ are shown as a function of $\sqrt{s_{p p}}$ by open stars in Fig. 2, along with the existing multiplicity measurements from $p p / \bar{p} p$ collisions.

The measured $N_{\mathrm{ch}}^{p p}$ dependence on $\sqrt{s_{p p}}$ in the energy range spanning the interval between a few $\mathrm{GeV}$ to $1.8 \mathrm{TeV}$ are fitted with the power-law, second-order log polynomial and the hybrid functions. The hybrid and the power-law fits read

$$
\begin{aligned}
N_{\mathrm{ch}}^{p p}= & (1.60 \pm 0.23)+(-0.03 \pm 0.10) \ln \left(s_{p p}\right) \\
& +(0.18 \pm 0.01) \ln ^{2}\left(s_{p p}\right)+(0.03 \pm 0.02) s_{p p}^{(0.29 \pm 0.06)},
\end{aligned}
$$

and

$N_{\mathrm{ch}}^{p p}=(-7.36 \pm 0.16)+(6.97 \pm 0.12) s_{p p}^{(0.133 \pm 0.001)}$,

correspondingly.

From Fig. 2, one can conclude that the available data do not give any preference to one or another fit function. This is similar to the pre-LHC observations where the power-law fit were found to be indistinguishable from the $\log ^{2}$ polynomial fit at $\sqrt{s_{p p}}>53 \mathrm{GeV}$ [7]. Interestingly, these two functions are also found to fit equally well the nonsingle diffractive midrapidity density, as obtained by CMS: cf. fits in [42] vs those in [38]. The fit functions start to deviate from each other at the c.m. energy above a few $\mathrm{TeV}$ but still not far one from another even at $\sqrt{s_{p p}} \sim 10 \mathrm{TeV}$. This may point to apparently no change in the multihadron production in $p p$ interactions up to the highest LHC energy, in contrast to a new regime possibly emerging at $\sqrt{s_{N N}} \approx 1 \mathrm{TeV}$ in heavy-ion collisions.

It is remarkable how well the PDE predictions on $N_{\mathrm{ch}}^{p p}$ at $\sqrt{s_{p p}}>2 \mathrm{TeV}$ follow the power-law fit made to the measurements at $\sqrt{s_{p p}} \leq 1.8 \mathrm{TeV}$. This and the aboveindicated "no change" in the hadroproduction in $p p$ collisions as soon as one moves to $\mathrm{TeV}$ energies are in an agreement with the prediction [2], which seems to be the only successful one for the midrapidity density in $p p$ collisions at $\sqrt{s_{p p}}=7 \mathrm{TeV}[16]$.

\section{MULTIPLICITY CENTRALITY DEPENDENCE}

In this section, we address the point whether the centrality dependence of the mean multiplicity from heavy-ion experiments is described by Eq. (4), similarly to the midrapidity pseudorapidity density in [1]. In Fig. 3, we show the $N_{\text {part }}$-dependence of $N_{\mathrm{ch}} /\left(N_{\text {part }} / 2\right)$. The data are taken from the measurements by the PHOBOS experiment at RHIC [19] and by the ALICE experiment at LHC [23]. The solid triangles show the estimations using Eq. (4). As above, in the case of the $\sqrt{s_{N N}}$-dependence, the rapidity densities $\rho_{p p}(0)$ and $\rho(0)$, and the multiplicity $N_{\mathrm{ch}}^{p p}$ are taken from the existing data [8] or, where not available, are calculated from the fits described above. According to the consideration developed here, $\rho(0)$ is taken at $\sqrt{s_{N N}}=$ $\varepsilon_{N N}$, and $\rho_{p p}(0)$ and $N_{\mathrm{ch}}^{p p}$ are taken at $\sqrt{s_{p p}}=3 \varepsilon_{N N}$.

One can see that the calculations, which are driven by the centrality-defined effective c.m. energy $\varepsilon_{N N}$, well reproduce the LHC data except slightly underestimating a couple of the most peripheral measurements. For the RHIC data, however, the difference between the calculations and the measurements is visible already for 


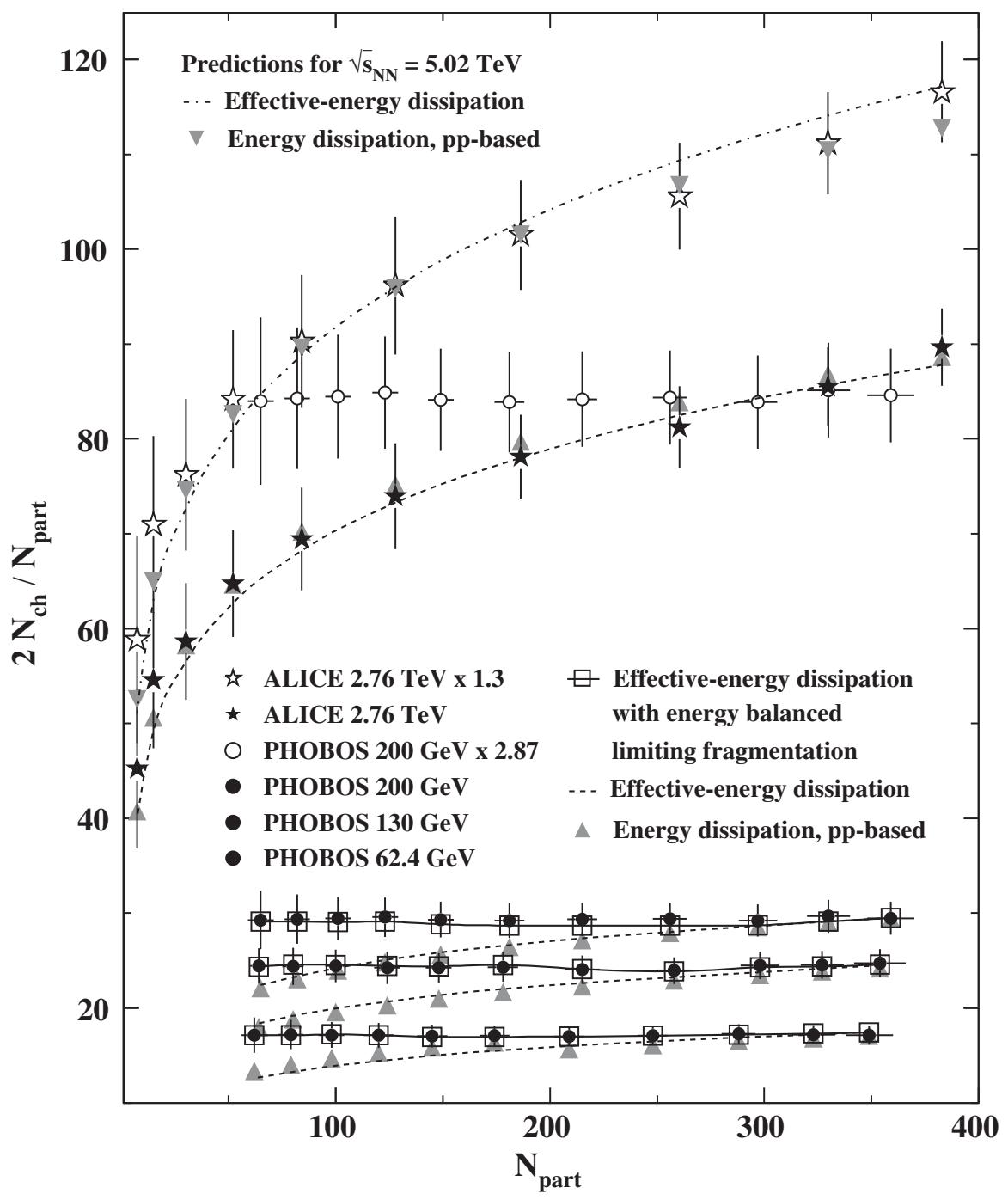

FIG. 3. The charged particle mean multiplicity per participant pair as a function of the number of participants, $N_{\text {part }}$. The solid circles show the dependence measured in AuAu collisions at RHIC by the PHOBOS experiment at $\sqrt{s_{N N}}=62.4,130$ and $200 \mathrm{GeV}$ [19] (bottom to top). The solid stars show the measurements from $\mathrm{PbPb}$ collisions at the LHC by the ALICE experiment at $\sqrt{s_{N N}}=$ $2.76 \mathrm{TeV}$ [23]. The triangles show the calculations by Eq. (4) using $p p / \bar{p} p$ data. The dashed lines represent the calculations within the effective-energy approach based on the hybrid fit, Eq. (5), to the c.m. energy dependence of the mean multiplicity in the most central heavy-ion collisions shown in Fig. 1. The dashed-dotted line show the predictions for $\sqrt{s_{N N}}=5.02 \mathrm{TeV}$ using the average of the fits, Eq. (5) and Eq. (6). The open squares show the effective-energy calculations which include the energy-balanced limiting fragmentation scaling (see text); the solid lines connect the calculations to guide the eye. The open circles show the PHOBOS measurements at $\sqrt{s_{N N}}=200 \mathrm{GeV}$ multiplied by 2.87. The open stars show the ALICE measurements at $\sqrt{s_{N N}}=2.76 \mathrm{TeV}$ multiplied by 1.3 .

medium centralities, i.e. for more central collisions. These observations are also interrelated with the difference observed in the measurements at RHIC vs those from LHC. Indeed, at RHIC, the participant-pair-normalized mean multiplicity is found to be independent of centrality, while a decrease with centrality, or monotonic increase with $N_{\text {part }}$, is observed at the LHC. This becomes even clearer when the $200 \mathrm{GeV}$ PHOBOS data are multiplied by a factor of 2.87 (open circles in Fig. 3) which allows matching the ALICE data from the highly central collisions.
In Fig. 3, the above-obtained c.m. energy fit, Eq. (5), made to the head-on collision data, is applied to the centrality measurements at $\sqrt{s_{N N}}=\varepsilon_{N N}$, and the results are shown by the dashed lines. ${ }^{4}$ The observations made for the calculations are valid here as well. This points to the complementarity of central collisions and centrality data once the calculations are made in the c.m. effective-energy $\varepsilon_{N N}$ terms.

To clarify the observed differences, in the following sections the distributions of the pseudorapidity density are

\footnotetext{
${ }^{4}$ Similar results are obtained from Eq. (6).
} 


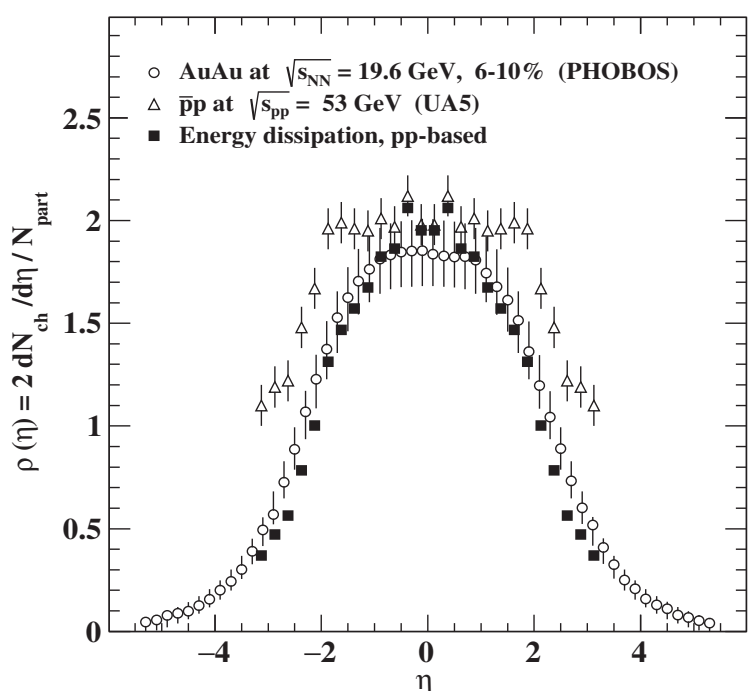

(a)

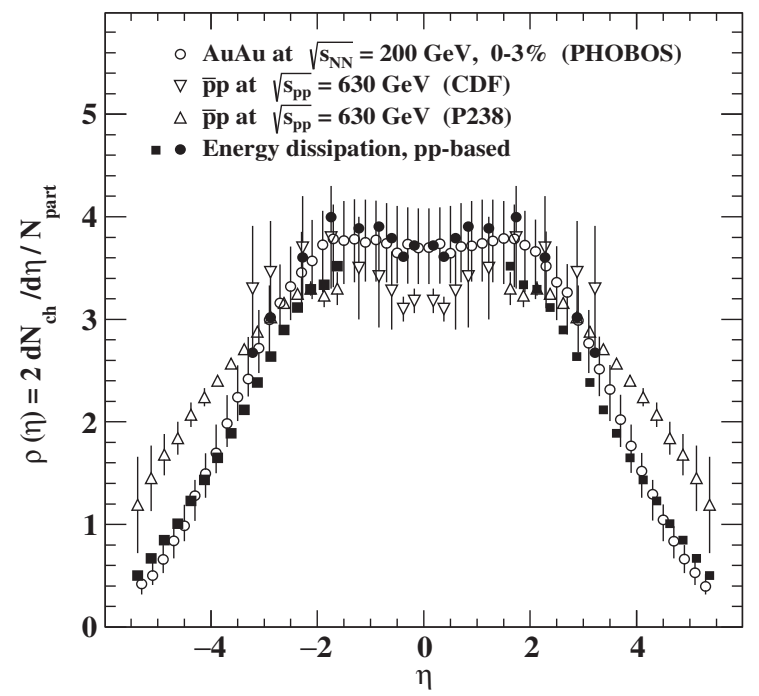

(c)

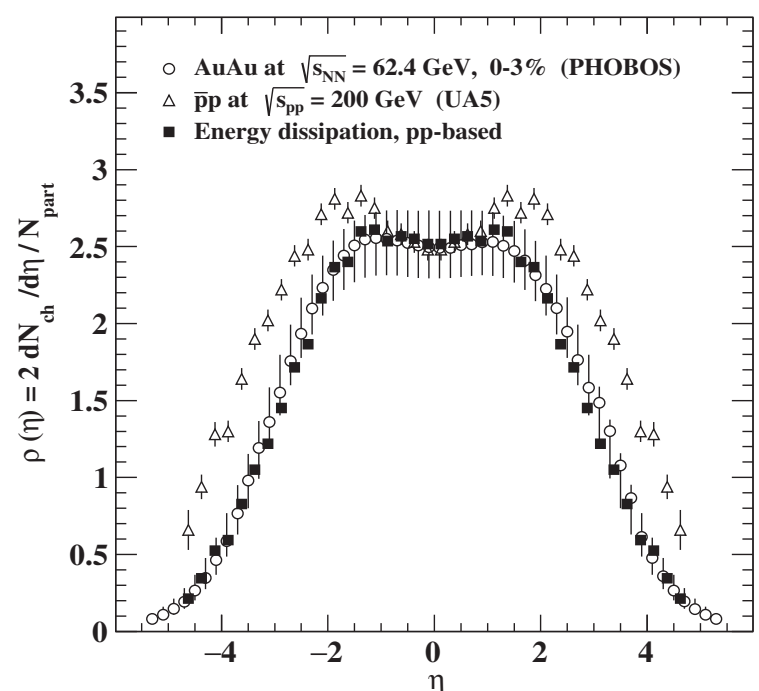

(b)

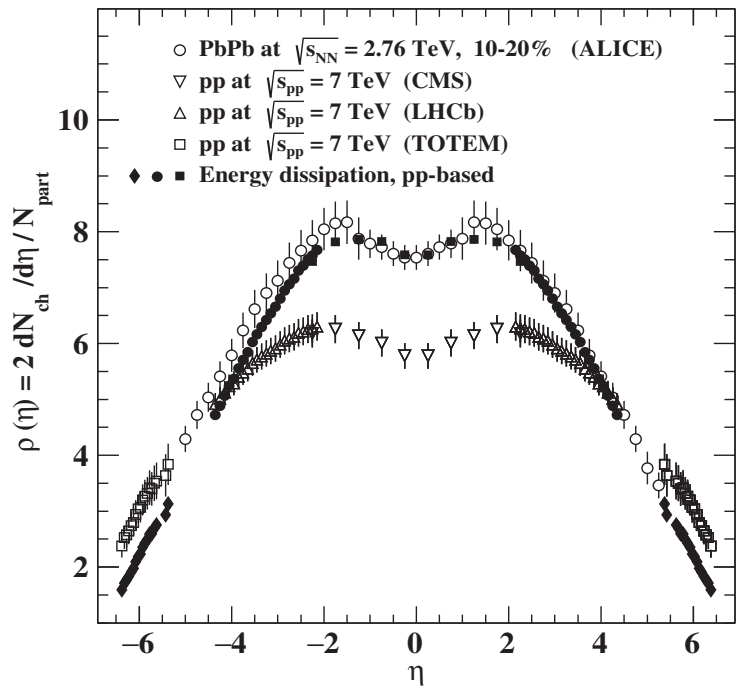

(d)

FIG. 4. The pseudorapidity distributions of charged particle pseudorapidity density per participant pair. The open circles show the measurements by the PHOBOS experiment in AuAu collisions at RHIC at (a) $\sqrt{s_{N N}}=19.6 \mathrm{GeV}$, (b) $62.4 \mathrm{GeV}$ and (c) $200 \mathrm{GeV}$ [19], and (d) by the ALICE experiment in PbPb collisions at the LHC at $\sqrt{s_{N N}}=2.76 \mathrm{TeV}$ [17]. The open triangles show the distributions measured in $\bar{p} p$ interactions by the UA5 experiment at $\sqrt{s_{p p}}=53 \mathrm{GeV}$ at the ISR and at $\sqrt{s_{p p}}=200 \mathrm{GeV}$ at the SPS [45], by the P238 experiment at the SPS [46] and by the CDF experiment at the Tevatron [47] at $\sqrt{s_{p p}}=630 \mathrm{GeV}$, and in $p p$ interactions by the CMS [42], LHCb [48] and TOTEM [49] experiments at $\sqrt{s_{p p}}=7 \mathrm{TeV}$ at the LHC. The solid markers show the calculations by Eq. (10) using $p p / \bar{p} p$ data at $\sqrt{s_{p p}} \approx 3 \sqrt{s_{N N}}$ or $3 \varepsilon_{N N}$. Apart from the CMS data, the negative- $\eta$ data points for $p p / \bar{p} p$ interactions are the reflections of the measurements taken in the positive- $\eta$ region.

investigated in the context of the PDE picture considered here.

\section{PSEUDORAPIDITY DENSITY DISTRIBUTION IN HEAVY-ION COLLISIONS: CENTRAL VS NONCENTRAL COLLISIONS}

Figure 4 shows the distributions of charged particle pseudorapidity density per pair of participants measured in head-on and very central heavy-ion collisions and in $p p / \bar{p} p$ interactions. The heavy-ion data represent the PHOBOS measurements made in $\mathrm{AuAu}$ collisions at the RHIC at $\sqrt{s_{N N}}=19.6,62.4$ and $200 \mathrm{GeV}$ [19] and the ALICE measurements from $\mathrm{PbPb}$ collisions at the LHC at $\sqrt{s_{N N}}=2.76 \mathrm{TeV}$ [17]. The distributions from $p p / \bar{p} p$ interactions are taken as measured by the UA5 experiment [45] at $\sqrt{s_{p p}}=53$ at the ISR and at $\sqrt{s_{p p}}=200 \mathrm{GeV}$ at the SPS, by the P238 experiment at the SPS [46] and by the $\mathrm{CDF}$ experiment at the Tevatron [47] at $\sqrt{s_{p p}}=630 \mathrm{GeV}$, 


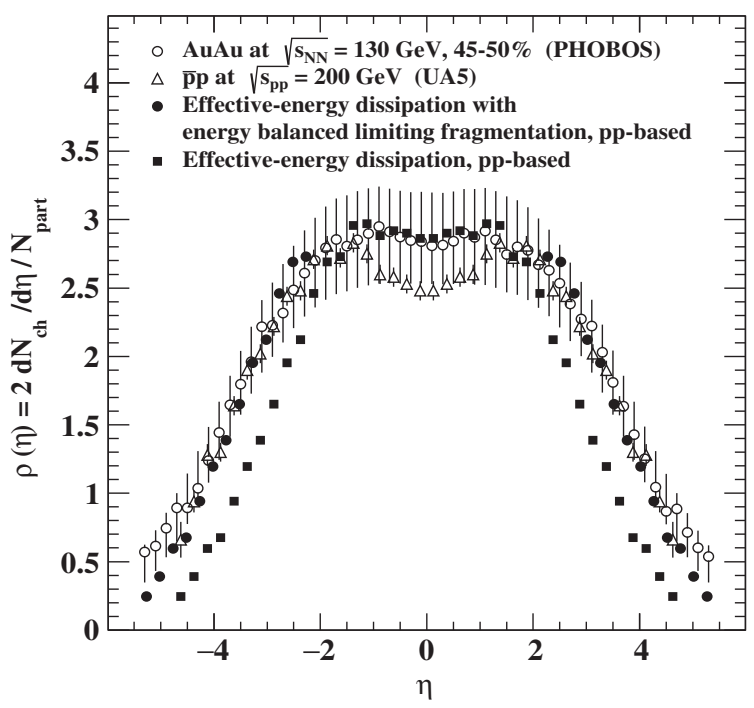

(a)

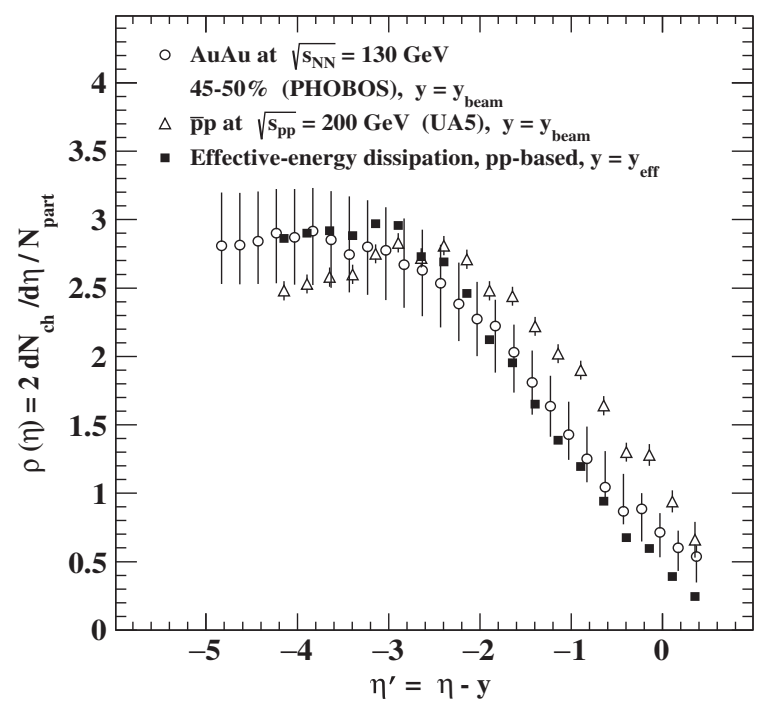

(b)

FIG. 5. (a) The charged particle pseudorapidity density per participant pair as a function of pseudorapidity. The open circles show the distribution measured in AuAu collisions at RHIC by the PHOBOS experiment at $\sqrt{s_{N N}}=130 \mathrm{GeV}$ in $45 \%-50 \%$ centrality interval. [19]. The open triangles show the distributions measured in $\bar{p} p$ interactions by the UA5 experiment at the SPS at $\sqrt{s_{p p}}=200 \mathrm{GeV}[45]$. The solid squares show the distribution calculated from Eq. (10) by using the UA5 $\bar{p} p$ data at $\sqrt{s_{p p}} \approx 3 \varepsilon_{N N}$ (see Eq. (3) for the definition of $\left.\varepsilon_{N N}\right)$. The solid circles show the beyond-midrapidity part obtained from the calculations using the energy-balanced limiting fragmentation scaling, i.e. under the shift $\eta \rightarrow \eta-\ln \left(\varepsilon_{N N} / \sqrt{s_{N N}}\right)$. The negative- $\eta$ data points for $\bar{p} p$ interactions are the reflections of the measurements taken in the positive- $\eta$ region. (b) Same as (a) but the measured distributions of AuAu and $\bar{p} p$ collisions are shifted by the beam rapidity, $\eta^{\prime}=\eta-y_{\text {beam }}$, with $y_{\text {beam }}=\ln \left(\sqrt{s} / m_{p}\right)$, where $s$ is, correspondingly, $s_{N N}$ or $s_{p p}$, and the calculated distribution is shifted to $\eta^{\prime}=\eta-y_{\text {eff }}$ with $y_{\text {eff }}=\ln \left(\varepsilon_{N N} / m_{p}\right)$. The distribution measured in AuAu collisions and the calculated distribution coincide in the fragmentation region, when being shifted by $y_{\text {beam }}$ for AuAu data and by $y_{\text {eff }}$ for the calculations, that represents the energy-balanced limiting fragmentation scaling.

and by the CMS [42], LHCb [48] and TOTEM [49] experiments at $\sqrt{s_{p p}}=7 \mathrm{TeV}$ at the LHC. The data shown are taken at the c.m. energies $\sqrt{s_{p p}} \approx 3 \sqrt{s_{N N}}$ or $3 \varepsilon_{N N}$. Except for the CMS measurements, the negative- $\eta$ data points from $p p / \bar{p} p$ interactions are the reflections of the measurements taken in the positive- $\eta$ region.

Within the considered model of constituent quarks and the Gaussian form of the pseudorapidity distribution in Landau hydrodynamics, the relationship between the pseudorapidity density distributions $\rho(\eta)$ and $\rho_{p p}(\eta)$ reads

$\frac{\rho(\eta)}{\rho_{p p}(\eta)}=\frac{2 N_{\mathrm{ch}}}{N_{\text {part }} N_{\mathrm{ch}}^{p p}} \sqrt{1+\frac{2 \ln 3}{L_{N N}}} \exp \left[\frac{-\eta^{2}}{L_{N N}\left(2+L_{N N} / \ln 3\right)}\right]$.

Here, all variables are defined the same way as in Eq. (1), i.e. taking into account the constituent quark scaling of the c.m. energy as soon as one relates $p p / \bar{p} p$ interactions to central heavy-ion collisions.

Using Eq. (10), the heavy-ion distributions are calculated based on the $\rho_{p p}(\eta)$ spectra shown in Fig. 4. The calculated distributions are shown by solid symbols in Fig. 4.

One can see that the calculations are in very good agreement with the measurements. Minor deviations are due to some mismatch between $\sqrt{s_{p p}}$ and $3 \sqrt{s_{N N}}$ (or $\left.3 \varepsilon_{N N}\right)$ and, as expected, due to a slight noncentrality; this is especially visible at $\sqrt{s_{N N}}=19.6 \mathrm{GeV}$ where the energy mismatch is of a largest fraction. It is noticeable how well the PDE picture allows one to reproduce the pseudorapidity density distributions from heavy-ion interactions in the full- $\eta$ range, from central- $\eta$ to forward- $\eta$ regions, in the $\sqrt{s_{N N}}$ range spanning over more than 2 orders of magnitude. Remarkably, the pseudorapidity density distributions, measured in $p p / \bar{p} p$ collisions, despite being above those measured in heavy-ion collisions at $19.6 \mathrm{GeV}$ or, on the contrary, lying far below the heavy-ion data from the LHC almost in the full- $\eta$ range, equally well reproduce the heavy-ion data as soon as being recalculated within the PDE approach. Interestingly, the calculations at the LHC energies, well reproduce the heavy-ion data despite the $p p$ measurements from the three different experiments are combined. A slight deviation in the negative- $\eta$ region is due to some asymmetry in the ALICE data.

Let us now address peripheral collisions to clarify the deviation in centrality dependence between the data and the calculations as it is observed in Fig 3.

In Fig. 5(a), the distribution $\rho(\eta)$ measured [19] in $\mathrm{AuAu}$ collisions by the PHOBOS experiment at 
$\sqrt{s_{N N}}=130 \mathrm{GeV}$ at $45 \%-50 \%$ centrality, $\alpha=0.475$, is shown along with the $\rho_{p p}(0)$ distribution measured in $\bar{p} p$ collisions by the UA5 experiment at $\sqrt{s_{p p}}=200 \mathrm{GeV}$ [45], i.e. at $\sqrt{s_{p p}} \approx 3 \varepsilon_{N N}$ according to our approach.

Applying Eq. (10), we calculate the $\rho(\eta)$ spectrum which is shown in Fig. 5(a) by solid squares. The calculations agree well with the measurements in the central- $\eta$ region while fall below the data outside this region. This finding shows that in noncentral collisions, the calculations within the approach, which combines the constituent quark picture and the relativistic Landau hydrodynamics, reproduces well the pseudorapidity density around the midrapidity while underestimate the mean multiplicity. The former conclusion is well confirmed by our recent studies reported in [1] for the midrapidity observables, and the latter one is demonstrated by Fig. 3 .

To clarify the obtained features, the following comments are due.

In the PDE picture proposed here, the global observables are defined by the energy of the participating constituent quarks pumped into the overlapped zone of the colliding nuclei. Hence, the bulk production is driven by the initial energy deposited at zero time at rapidity $\eta=0$, similar to the Landau hydrodynamics. Then, as is expected and mentioned above, the pseudorapidity density at midrapidity is well reproduced for all types of nuclear collisions, from the most central to peripheral ones. As shown in [1], similarly, the centrality dependence of the transverse energy density at midrapidity is well reproduced by the calculations and complements the c.m. energy dependence of the head-on data. Note that this similarity in the pseudorapidity density and the transverse energy pseudorapidity density is in accordance with the same functional form of the (pseudo)rapidity density distribution obtained either in the longitudinally expanding system considered in the original Landau model or when the development in the transverse direction is included [50-52].

From Fig. 5(a), one can see that the calculated distribution $\rho(\eta)$ is narrower than that of the data. The narrowness of the calculated distribution with respect to the measured one is explained by a smaller value of $\varepsilon_{N N}$ compared to the value of the actual collision energy $\sqrt{s_{N N}}$. However, the calculations in Eq. (10) are made with the multiplicity $N_{\mathrm{ch}}$ taken from the most central nucleusnucleus collisions at the c.m. energy equal to $\varepsilon_{N N}$ (and similarly in Eqs. (2) and (4) for the midrapidity density $\rho(0)$ ). In other words, in the approach applied here, similar to the Landau hydrodynamics, the collisions of nuclei are treated head-on-like.

\section{ENERGY-BALANCED LIMITING FRAGMENTATION}

It is established that at high enough energies, in different types of interactions the pseudorapidity density spectra, measured at different c.m. energies, become similar in the fragmentation region. It means that they are independent of a projectile state (which is the beam or target rest frame) for the same type of colliding objects, i.e. being considered as a function of $\eta^{\prime}=\eta-y_{\text {beam }}$, where $y_{\text {beam }}=\ln \left(\sqrt{s_{N N}} / m_{p}\right)$ is the beam rapidity $[6,10]$. This observation obeys a hypothesis of the limiting fragmentation scaling [53].

Considering the limiting fragmentation hypothesis within the effective-energy approach, one expects the limiting fragmentation scaling of the distribution $\rho(\eta)$, which is measured at $\sqrt{s_{N N}}$, to be similar to that of the calculated distribution but taken at the effective energy $\varepsilon_{N N}$. Note that the limiting fragmentation phenomenon, though being expected as an universal phenomenon for the Gaussian form of $\rho(\eta)[10,54,55]$, naturally arises in Landau hydrodynamics [9].

In Fig. 5(b), the limiting fragmentation hypothesis is applied to both the measured and the calculated pseudorapidity density distributions $\rho(\eta)$ from Fig. 5(a) using the c.m. energy and the effective energy, respectively. Therefore, the measured distribution $\rho(\eta)$ is shifted by the beam rapidity, $y_{\text {beam }}$, while the calculated distribution from Eq. (10) is shifted by $y_{\text {eff }}=\ln \left(\varepsilon_{N N} / m_{p}\right)$ and becomes a function of $\eta^{\prime}=\eta-y_{\text {eff }}$, as expected. One can see that the calculated $\rho\left(\eta^{\prime}\right)$ distribution of noncentral heavy-ion collisions agrees well with the measured distribution $\rho\left(\eta^{\prime}\right)$. This finding points to a new energy scaling as soon as the effective-energy approach is applied. In analogy with the limiting fragmentation scaling, we call the observed scaling the "energy-balanced limiting fragmentation scaling." Due to this scaling, the calculated pseudorapidity density is getting corrected outside the central- $\eta$ region accordingly.

To this end, in Fig. 5(a), the calculated distribution $\rho(\eta)$ is shifted by the difference $\left(y_{\text {eff }}-y_{\text {beam }}\right)$ in this region: $\eta \rightarrow \eta-\ln \left(\varepsilon_{N N} / \sqrt{s_{N N}}\right)$, or, using the effective-energy definition, Eq. (3), $\eta \rightarrow \eta-\ln (1-\alpha)$. The calculated distribution $\rho(\eta)$, where the shift is applied, is shown by the solid circles in Fig. 5(a). The shift balances the energy and this brings the calculations to the measured pseudorapidity density distribution in the full- $\eta$ range in noncentral heavyion collision. It is clear that in head-on or very central collisions, $\alpha$ approaches zero which makes the shift negligible (cf. Fig. 4).

This finding allows obtaining $N_{\text {ch }}$ within the PDE approach. Namely, the difference between the two $N_{\text {ch }}$ values, one obtained by integrating the calculated pseudorapidity density distribution from Eq. (10), and another one of the same distribution but being shifted to the left by $\ln (1-\alpha)$, is added to the $N_{\text {ch }}$ value obtained from Eq. (4). Where no pseudorapidity density distributions are available in $p p / \bar{p} p$ measurements at $\sqrt{s_{p p}}=3 \varepsilon_{N N}$, the energybalanced limiting fragmentation scaling is applied to reproduce the calculated $\rho(\eta)$ : the measured distribution from a noncentral heavy-ion collision is shifted by $\left(y_{\text {beam }}-y_{\text {eff }}\right)$, i.e. $\eta \rightarrow \eta+\ln (1-\alpha)$. Then $N_{\text {ch }}$ is 
calculated as above, by adding to the calculation of Eq. (4) the difference between the integral from the obtained shifted distribution and the measured multiplicity in this noncentral heavy-ion collision.

Using this ansatz, the values of $N_{\mathrm{ch}}$ are calculated for each centrality for the RHIC measurements. The calculations are shown by open squares in Fig. 3. One can see that now the calculations well reproduce the measurements from RHIC, with no deficit in noncentral collisions.

The energy-balanced limiting fragmentation scaling provides an explanation of the "puzzle" between the centrality independence of the $N_{\text {part }}$-normalized mean multiplicity and the monotonic decrease of the normalized midrapidity pseudorapidity density with the centrality, as observed at RHIC. As shown above, the pseudorapidity density at midrapidity is determined by the effective energy of centrally colliding nucleon participants. Hence, the value of this observable increases towards head-on collisions as soon as the effective energy, made available for particle production, increases with increasing number of participants (decreasing centrality). However, the multiplicity is measured in the full $\eta$-region, so it gets additional contribution from beyond the midrapidity. In the context of the PDE picture, this contribution is due to the balance between the collision c.m. energy shared by all nucleons of colliding nuclei and the centrality-defined effective energy of the interacting participants. The more peripheral is the collision, the larger the additional contribution is. This contribution can be directly estimated by the energy-balanced limiting fragmentation scaling, introduced here, which leads to the scaling between the measured pseudorapidity distribution and the distribution calculated within the PDE approach.

From Fig. 3 one can conclude that, in contrast to the RHIC measurements, almost no additional contribution is needed for the PDE calculations of Eq. (4) in order to describe the LHC mean multiplicity data. As the calculations imply, they are made by considering the nucleusnucleus collisions as head-on collisions at the c.m. energy of the value of $\varepsilon_{N N}\left(\rho(0)\right.$ in Eq. (4) as well as $N_{\text {ch }}$ in Eq. (10) are taken from the head-on $\sqrt{s_{N N}}$ fits). However, as shown above, the additional contribution to the mean multiplicity increases with increasing collision centrality, i.e. while going towards more peripheral collisions. For head-on collisions, however, this contribution tends to zero. Given the multiplicity measurements at the LHC are well reproduced without the energy-balanced additional contribution, one concludes that in heavy-ions collisions at the $\mathrm{LHC}$ at $\mathrm{TeV}$ energies the multihadron production obeys a head-on collision regime, for all the centrality intervals measured. This points to apparently different regimes of hadroproduction occurring in heavy-ion collisions with $\sqrt{s_{N N}}$ between a few hundred $\mathrm{GeV}$ and $\mathrm{TeV}$ energies. This observation supports a similar conclusion made above, which is suggested from the observation of a change of the functional type of the fit needed to describe the energy behavior as soon as the LHC data are included, see Fig. 1.

The discussed difference between the mean multiplicity, and hence the full pseudorapidity density distributions, measured in heavy-ion collisions at RHIC and at LHC have been earlier addressed in [32,37], where the model of three sources, the gluon-gluon midrapidity and two quark-gluon fragmentation sources, are applied to understand the observations from experimental data. In the context of the PDE approach given here, the difference in the nature of collisions at effective c.m. energy is appealed to explain different centrality dependence of the data from the two colliders. Similarly to calculations in [32,37], additional contribution from the fragmentation regions are shown to be needed at RHIC. However, no such contribution is needed at the LHC energy. Meantime, the midrapidity pseudorapidity densities measured at RHIC and at LHC do not show different behavior with centrality and are found [1] to be similarly well reproduced by the PDE calculations where no preference is given to midrapidity or fragmentation sources.

There are other approaches, which also consider the three effective regions in pseudorapidity density distributions of charged particles produced in $p p / \bar{p} p$ and in heavyion collisions. In the string percolation model $[36,56]$, the fragmentation region is populated by strings of valence quarks and the midrapidity region by additional short strings between quarks and antiquarks. In other approaches, one introduces a leading particle activity within the hydrodynamic [57-60] or thermal [61] pictures of the multiparticle production processes. Like in the PDE consideration, presented here, a similarity of the mechanism of particle production in $p p / \bar{p} p$ and heavy-ion collisions is also assumed in these approaches. Whereas within the PDE approach the leading particles resulting from the spectators are considered to be produced in nucleon-nucleon collisions, where a single quark pair interaction is assumed, no leading particle effect is implied for central nucleus-nucleus collisions, where the entire energy of the participants is considered to be available for bulk hadron production. As already noticed above, no difference in the particle production sources in different pseudorapidity regions is assumed in the PDE approach. Then, the c.m. energy scaling due to the key picture of the constituent quarks, applied to the Landau hydrodynamics, allows revealing the universality of the multihadron dynamics in hadronic and nuclear interactions.

\section{MULTIPLICITY EFFECTIVE ENERGY DEPENDENCE IN HEAD-ON AND NONCENTRAL COLLISIONS}

Given the obtained agreement between the data and the calculations, and considering the similarity put forward for $\varepsilon_{N N}$ and $\sqrt{s_{N N}}$, one would expect the measured centrality data at $\varepsilon_{N N}$ to follow the $\sqrt{s_{N N}}$ dependence of the mean 
multiplicity in the most central nuclear collisions. In Fig. 1, the measurements of the charged particle mean multiplicity of head-on nuclear collisions are added by the centrality measurements by the PHOBOS [19] and the ALICE [23] experiments (Fig. 3) where the centrality data are plotted as a function of $\varepsilon_{N N}$. Due to the above finding of the energybalanced limiting fragmentation scaling, explaining the lack of centrality dependence of the mean multiplicity at RHIC energies, these data are plotted by subtracting the energy-balanced contribution. In addition, the centrality data at $\sqrt{s_{N N}}=19.6 \mathrm{GeV}$ are shown in Fig. 1 but not in Fig. 3. From Fig. 1, one concludes that effective-energy dependence of the centrality data complements the c.m. energy behavior of the head-on collision data.

To better trace the similarity between the head-on collision and centrality data, we fit the $\varepsilon_{N N}$-dependence of the centrality data by the hybrid and the power-law functions, similarly to the head-on collisions. For the hybrid fit, one gets

$$
\begin{aligned}
\frac{2 N_{\mathrm{ch}}}{N_{\text {part }}}= & (3.04 \pm 0.60)-(1.40 \pm 0.24) \ln \left(\varepsilon_{N N}\right) \\
& +(1.12 \pm 0.04) \ln ^{2}\left(\varepsilon_{N N}\right) \\
& +(0.032 \pm 0.028) \varepsilon_{N N}^{(0.848 \pm 0.106)}
\end{aligned}
$$

This fit is shown by the dashed line in Fig. 1. This fit agrees well with the same type of the fit to the head-on collision data in the entire available energy range though lying slightly above the latter one for the data at $\sqrt{s_{N N}} \lesssim 10 \mathrm{GeV}$. For the $\log ^{3}\left(\varepsilon_{N N}\right)$ fit function of the three-sources approach, similar to Eq. (7), one finds

$$
\begin{aligned}
\frac{2 N_{\mathrm{ch}}}{N_{\mathrm{part}}}= & (1.70 \pm 1.49)+(1.18 \pm 0.54) \ln \left(\varepsilon_{N N}\right) \\
& +(0.152 \pm 0.008) \ln ^{3}\left(\varepsilon_{N N}\right) .
\end{aligned}
$$

The fit is shown by the thin dashed-dotted line in Fig. 1, and lies on top of the analogous fit, Eq. (7) to the head-on data, except a slight enhancement at $\sqrt{s_{N N}} \lesssim 10 \mathrm{GeV}$, similar to the hybrid fit. The power-law $\varepsilon_{N N}$-fit for the centrality data is shown by the dotted line in Fig. 1. It is found to be similar to the power-law $\sqrt{s_{N N}}$-fit, Eq. (6), to the head-on collision data shown by the dashed-dotted line.

From this one concludes that within the picture proposed here the data are well reproduced under the assumption of the effective energy which governs the multiparticle production. This points to the same energy behavior in multihadron production for all types of heavy-ion collisions, from peripheral to the most central collisions.

Here, let us stress an important corollary of the PDE approach. As soon as the effective energy in nucleusnucleus collisions determines the pseudorapidity density at midrapidity, then the midrapidity pseudorapidity densities at the same effective energy but at different c.m. energy get the same value. In other words, the densities are defined by the effective energy independent of the energy of the collision. The observation made here for the multiplicity dependence on the effective energy confirms the observation made earlier for the midrapidity densities [1], while it adds another important ingredient which takes into account the additional energy-balanced contribution to the mean multiplicity in noncentral nucleus-nucleus collisions.

From the hybrid fits obtained, we estimate the multiplicity for the future LHC heavy-ion run. Since the hybrid fit for the head-on collision data and the fit to the centrality data show a slightly different increase with c.m. energy, the predictions of the two fits are averaged. Hence, the mean multiplicity $2 N_{\mathrm{ch}} / N_{\text {part }}$ value is predicted to be about 119 with $5 \%$ uncertainty in the most central heavy-ion collisions at $\sqrt{s_{N N}}=5.13 \mathrm{TeV}$. The prediction is shown by the right-inclined hatched area in Fig. 1. This value is close to the value of about 116 one gets from the ALICE fit [17] and about 108 which one obtains from Eq. (7). In addition, the fit-averaged prediction based on $p p$ collisions at $\sqrt{s_{p p}}=13 \mathrm{TeV}$, recalculated within the PDE approach, is shown in Fig. 1 as the left-inclined hatched area.

The predictions are made as well for the centrality dependence and are given in Fig. 3. We give the predictions for $\sqrt{s_{N N}}=5.02 \mathrm{TeV}$, considering the recent measurements reported by ALICE for the rapidity density [39] and expecting the mean multiplicity measurements at this energy. The two types of predictions are shown.

First, similar to the above predictions made to the headon collisions, we use the fit functions. As soon as, within the effective-energy approach, we treat noncentral collisions as central collisions at energy $\varepsilon_{N N}$, then we use the head-on collision multiplicity fits to predict the centrality dependence. This is similar to that made for the existing data, as shown in Fig. 3 by the dashed lines. However, for the predictions, we use the average values of the hybrid and the power-law fits, Eqs. (5) and (6), as soon as those deviate for $\sqrt{s_{N N}}$ above $2.76 \mathrm{TeV}$. The prediction for $\sqrt{s_{N N}}=$ $5.13 \mathrm{TeV}$ centrality dependence is shown by the dasheddotted line. The centrality and $N_{\text {part }}$ values are alike in the $2.76 \mathrm{TeV}$ data shown. The expectations show an increase of the mean multiplicity with $N_{\text {part }}$ (decrease with centrality) from about 52 to about 118 . The increase looks to be slightly faster than at $\sqrt{s_{N N}}=2.76 \mathrm{TeV}$, especially for the peripheral region.

Second, the PDE set of prediction is made using the calculations based on Eq. (4) combining the constituent quark model and the Landau hydrodynamics. This prediction for the centrality dependence is shown by the solid inverted triangles. One can see that the predictions are close to ones obtained from the head-on collision data fits.

The predictions are compared with the LHC data at $\sqrt{s_{N N}}=2.76 \mathrm{TeV}$. To better match the predictions for highly central collisions, the $2.76 \mathrm{TeV}$ data points are 
multiplied by 1.3. One can see that the predictions are well reproduced by the scaled data. This indicates no change of the hadroproduction mechanism expected with increase of the c.m. energy at LHC, in contrast to what is seen as one compares the scaled $200 \mathrm{GeV}$ RHIC data with the $2.76 \mathrm{TeV}$ measurements.

An interesting issue to be addressed in the framework of the PDE picture is asymmetric collisions, such as nucleonnucleus ( $p / d$-nucleus) ones. In these interactions, the multiplicity is also expected to have no centrality dependence. This is due to the many nucleon-nucleon interactions of the incident proton with the nucleons of the interacting nucleus while the secondary particles produced in the reaction are assumed to be created out of the c.m. energy deposited to the interaction zone. The proton and nucleus are considered to interact via a single pair of constituent quarks, one from the proton and another one from a nucleon in the interacting nucleus. Then, no centrality dependence of the multiplicity is expected in $p$-nucleus collisions with the multiplicity values to be similar to that from $p p / \bar{p} p$ interactions at the c.m. energy $\sqrt{s_{p p}} \simeq \sqrt{s_{N N}}$. As a consequence, this, at a given centrality, results in $N_{\text {part }} / 2$ for the ratio $N_{\mathrm{ch}} / N_{\mathrm{ch}}^{p p}$. These features have been indeed obtained in $d$-Au interactions at RHIC [62]. Moreover, the effect of the $N_{\text {part }}$-dependence of the multiplicity ratio obtained at RHIC has been also observed in hadron-nucleus collisions at lower $\sqrt{s_{N N}} \approx 10-20 \mathrm{GeV}$ $[62,63]$. These observations seem to be also obtained at LHC, where the dependence of the pseudorapidity density on $\sqrt{s_{N N}}$ measured in $p-\mathrm{Pb}$ collisions at $\sqrt{s_{N N}}=5.02 \mathrm{TeV}$ is observed [64] to be consistent with a power-law $\sqrt{s_{p p}}$-fit to $p p / \bar{p} p$ data and its centrality dependence is shown $[65,66]$ to demonstrate the importance of nucleon-nucleon interactions for $p-\mathrm{Pb}$ results.

\section{SUMMARY AND CONCLUSIONS}

In summary, the multihadron production process in nucleus-nucleus collisions and its universality in nuclear and hadronic interactions are studied. The study exploits the charged particle mean multiplicity dependencies on the c.m. collision energy per nucleon, $\sqrt{s_{N N}}$, and on the number of nucleon participants, or centrality, measured in the energy range of a few $\mathrm{GeV}$ to a few $\mathrm{TeV}$. The study is carried out in the framework of the earlier proposed approach of the dissipating effective energy of constituent quark participants $[2,3]$, or the participant dissipating energy (PDE) approach. In this approach, the participants are considered to form the initial zone of a collision and to determine the production of hadrons at the very early stage of the collision. In this consideration, one combines the constituent quark picture with Landau hydrodynamics and interrelates the multihadron production in different types of collisions by a proper scaling of the c.m. energy of collisions. In particular, an energy-scaling factor of $1 / 3$ in $p p / \bar{p} p$ measurements is shown to reveal the universality of the multiplicity dependencies in nucleon-nucleon and nucleus-nucleus interactions.

In the entire available $\sqrt{s_{N N}}$ range of about a few $\mathrm{TeV}$, the energy dependence of the multiplicity in head-on collisions is found to be well described by the calculations performed within the effective-energy approach based on $p p / \bar{p} p$ data. Meanwhile, depending on the data sample, the calculations are found either to describe the measured centrality dependence or to show some deviation between the calculations and the data. For the RHIC data, the deficit in the predictions is observed for noncentral collisions so that the predictions do not follow a constancy with the centrality as it is observed at RHIC. The LHC mean multiplicity centrality dependence, however, is found to be well described by the calculations including the increase towards the most central collisions.

To clarify the observations, approach of the effective energy of the quark participants is applied to the pseudorapidity density distribution measured in heavy-ion collisions. The energy-balanced limiting fragmentation scaling is introduced based on assumption of the similarity of the fragmentation region of the measured distribution in the beam rest frame and that determined from the calculations by using the effective energy. The revealed scaling allows us to reproduce the pseudorapidity density distributions independently of the centrality of collisions and then to correctly describe the centrality independence of the mean multiplicity measured at RHIC. Moreover, this finding provides a solution to the RHIC "puzzle" of the difference between the centrality independence of the mean multiplicity vs the monotonic decrease of the midrapidity pseudorapidity density with the increase of centrality. The mean multiplicity is shown to get a fraction of additional contribution to account for the balance between the collision c.m. energy shared by all nucleons and the effective energy of the participants. However, the midrapidity pseudorapidity density is fully defined by the effective energy of colliding participants.

Given that the calculations made in the context of the proposed approach are considering central collisions of nuclei, an agreement between the calculations and the LHC data indicates that, at $\mathrm{TeV}$ energies, the collisions seem to present head-on collisions of the participants at the c.m. energy of the scale of the effective energy. Thus, no energybalanced additional contribution is required even with a relatively small number of participants at $\mathrm{TeV}$ energies.

Based on the above findings, the complementarity of the head-on collisions and the centrality data is shown, resulting in the similar energy behavior of the mean multiplicity measurements as soon as the data are considered in terms of the effective energy. A departure of the c.m. energy dependence of the data from the second-order logarithmic behavior to the power-law or higher-order logarithmic polynomial function one observes at the region of $1 \mathrm{TeV}$ 
suggests a transition to a new regime in nucleus-nucleus collisions at $\mathrm{TeV}$ energies. Interestingly, these findings made for a full collision rapidity range are similar to those drawn from the studies [1] of the pseudorapidity density and the transverse energy density at midrapidity. This is also in accordance with the change of the multiplicity dependence on centrality which also indicates a possible change of the regime of multihadron production as one moves from the RHIC to LHC energies.

The hybrid and the power-law fits are found to describe well the existing data on the charged particle multiplicity from $p p / \bar{p} p$ interactions in the entire c.m. energy $\sqrt{s_{p p}}$ range up to the top Tevatron energy of $1.8 \mathrm{TeV}$. However, in this case, no clear change from the power-law behavior to the quadratic log polynomial one is obtained in the multiplicity c.m. energy dependence. Moreover, the predictions made here for the mean multiplicity for $\sqrt{s_{p p}}$ in the LHC energy range of 2.36 to $13 \mathrm{TeV}$ within the PDE approach demonstrate a closeness between the predicted values and the lower-energy $\sqrt{s_{p p}}$ fit. One concludes that, in contrast to heavy ions, no change in multihadron production in $p p$ collisions is expected up to the foreseen LHC energy.

Based on the results of the hybrid fits, the predictions for the charged particle mean multiplicity in head-on heavy-ion collisions at $\sqrt{s_{N N}}=5.13 \mathrm{TeV}$ at the LHC are given. Within the obtained complementarity of head-on collisions and centrality data, the predictions are made for the mean multiplicity centrality dependence to be measured.

The soon-to-come measurements at the LHC are of crucial importance for further understanding of the multihadron dynamics. This will shed the light on the universality of the multihadron production process in different types of collisions and clarify the PDE approach and the obtained energy-balanced limiting fragmentation which have been shown to successfully describe the features of global key observables by relating hadronic and nuclear collisions.

\section{ACKNOWLEDGMENTS}

Thanks go to Lev Kheyn for his interest in the work and enlightening discussions. We are grateful to Gideon Alexander, Amarjeet Nayak, David Plane, Vladimir Roinishvili and Marek Taševský for their help during the work on this paper. The work of Alexander Sakharov is partially supported by the U.S. National Science Foundation under Grants No. PHY-1205376 and No. PHY-1402964.
[1] A. N. Mishra, R. Sahoo, E. K. G. Sarkisyan, and A. S. Sakharov, Eur. Phys. J. C 74, 3147 (2014).

[2] E. K. G. Sarkisyan and A. S. Sakharov, Eur. Phys. J. C 70, 533 (2010).

[3] E. K. G. Sarkisyan and A. S. Sakharov, AIP Conf. Proc. 828, 35 (2003).

[4] P. V. Chliapnikov and V. A. Uvarov, Phys. Lett. B 251, 192 (1990).

[5] B. B. Back et al. (PHOBOS Collaboration), arXiv:nucl-ex/ 0301017; Phys. Rev. C 74, 021902 (2006).

[6] W. Kittel and E. A. De Wolf, Soft Multihadron Dynamics (World Scientific, Singapore, 2005).

[7] J. F. Grosse-Oetringhaus and K. Reygers, J. Phys. G 37, 083001 (2010).

[8] K. A. Olive et al. (Particle Data Group), Chin. Phys. C 38, 090001 (2014).

[9] L. D. Landau, Izv. Akad. Nauk: Ser. Fiz. 17, 51 (1953) [English translation: Collected Papers of L. D. Landau, edited by D. Ter-Haarp (Pergamon, Oxford, 1965), p. 569; Reprinted in: Quark-Gluon Plasma: Theoretical Foundations, edited by J. Kapusta, B. Müller, and J. Rafelski (Elsevier, Amsterdam, 2003), p. 283.]

[10] See e.g. P. Steinberg, J. Phys. Conf. Ser. 9, 280 (2005).

[11] E. M. Levin and L. L. Frankfurt, JETP Lett. 2, 65 (1965).

[12] H. J. Lipkin and F. Scheck, Phys. Rev. Lett. 16, 71 (1966).
[13] J. J. J. Kokkedee and L. Van Hove, Nuovo Cimento 42, 711 (1966).

[14] For review and a collection of reprints on original papers on quarks and composite models, including, see J. J. J. Kokkedee, The Quark Model (W. A. Benjamin, Inc., New York, 1969).

[15] V. V. Anisovich, N. M. Kobrinsky, J. Nyiri, and Yu. M. Shabelsky, Quark Model and High Energy Collisions (World Scientific, Singapore, 2004).

[16] See e.g. R. Rougny (for the CMS Collaboration), Nucl. Phys. B, Proc. Suppl. 207-208, 29 (2010).

[17] E. Abbas et al. (ALICE Collaboration), Phys. Lett. B 726, 610 (2013).

[18] B. B. Back et al. (PHOBOS Collaboration), Nucl. Phys. 757, 28 (2005).

[19] B. Alver et al., Phys. Rev. C 83, 024913 (2011).

[20] S. V. Afanasiev et al. (NA49 Collaboration), Phys. Rev. C 66, 054902 (2002).

[21] J. L. Klay (E895 Collaboration), Ph.D. Thesis, U.C. Davis, 2001; see [18].

[22] G. Agakishev et al. (HADES Collaboration), Eur. Phys. J. A 40, 45 (2009).

[23] J. Adam et al. (ALICE Collaboration), Phys. Lett. B 754, 373 (2016).

[24] C. S. Lindsey, Nucl. Phys. A544, 343 (1992). 
[25] G. J. Alner et al. (UA5 Collaboration), Phys. Rep. 154, 247 (1987).

[26] R. E. Ansorge et al. (UA5 Collaboration), Z. Phys. C 43, 357 (1989).

[27] A. Breakstone et al., Phys. Rev. D 30, 528 (1984).

[28] W. M. Morse, V. E. Barnes, D. D. Carmony, R. S. Christian, A. F. Garfinkel, L. K. Rangan, A. R. Erwin, E. H. Harvey, R. J. Loveless, and M. A. Thompson, Phys. Rev. D 15, 66 (1977).

[29] V. V. Ammosov et al., Phys. Lett. 42B, 519 (1972).

[30] C. Bromberg et al., Phys. Rev. Lett. 31, 1563 (1973).

[31] E. De Wolf, J. J. Dumont, and F. Verbeure, Nucl. Phys. B87, 325 (1975).

[32] G. Wolschin, J. Phys. G 40, 045104 (2013).

[33] R. Sahoo, A. N. Mishra, N. K. Behera, and B. K. Nandi, Adv. High Energy Phys. 2015, 612390 (2015).

[34] E. Levin and A.H. Rezaeian, Phys. Rev. D 83, 114001 (2011).

[35] A. Capella and E. G. Ferreiro, Eur. Phys. J. C 72, 1936 (2012).

[36] I. Bautista, J. G. Milhano, C. Pajares, and J. Dias. de Deus, Phys. Lett. B 715, 230 (2012).

[37] G. Wolschin, Phys. Rev. C 91, 014905 (2015).

[38] S. Chatrchyan et al. (CMS Collaboration), J. High Energy Phys. 08 (2011) 141.

[39] J. Adam et al. (ALICE Collaboration), arXiv: 1512.06104.

[40] V. Khachatryan et al. (CMS Collaboration), J. High Energy Phys. 02 (2010) 041.

[41] K. Aamodt et al. (ALICE Collaboration), Eur. Phys. J. C 68, 89 (2010).

[42] V. Khachatryan et al. (CMS Collaboration), Phys. Rev. Lett. 105, 022002 (2010).

[43] G. Aad et al. (ATLAS Collaboration), New J. Phys. 13, 053033 (2011).

[44] J. Benecke et al., Nucl. Phys. 76, 29 (1974).

[45] G. J. Alner et al. (UA5 Collaboration), Z. Phys. C 33, 1 (1986).
[46] R. Haar et al. (P238 Collaboration), Phys. Lett. B 401, 176 (1997).

[47] F. Abe et al. (CDF Collaboration), Phys. Rev. D 41, 2330 (1990).

[48] R. Aaij et al. (LHCb Collaboration), Eur. Phys. J. C 72, 1947 (2012).

[49] G. Antchev (TOTEM Collaboration), Europhys. Lett. 98, 31002 (2012).

[50] G. A. Milekhin, Sov. Phys. JETP 8, 829 (1959).

[51] E. V. Shuryak, Sov. J. Nucl. Phys. 20, 295 (1975).

[52] E. L. Feinberg, in Relativistic Heavy Ion Physics, edited by L. P. Csernai and D. D. Strottman, International Review of Nuclear Physics Vol. 6 (World Scientific, Singapore, 1991), p 341.

[53] J. Benecke, T. T. Chou, C. N. Yang, and E. Yen, Phys. Rev. 188, 2159 (1969).

[54] C.-Y. Wong, Phys. Rev. C 78, 054902 (2008).

[55] A. Sen, J. Gerhard, G. Torrieri, K. Read, and C.-Y. Wong, Phys. Rev. C 91, 024901 (2015).

[56] I. Bautista, C. Pajares, J. G. Milhano, and J. Dias de Deus, Phys. Rev. C 86, 034909 (2012).

[57] Z. J. Jiang and H. L. Zhang, Mod. Phys. Lett. A 29, 1450130 (2014).

[58] Z. J. Jiang, H. L. Zhang, J. Wang, and K. Ma, Adv. High Energy Phys. 2014, 248360 (2014).

[59] Jiang, Y. Zhang, H. L. Zhang, and H. P. Deng, Nucl. Phys. 941, 188 (2015).

[60] L.-N. Gao and F.-H. Liu, Adv. High Energy Phys. 2015, 184713 (2015).

[61] B.-C. Li, Y.-Z. Wang, F.-H. Liu, X.-J. Wen, and Y.-E. Dong, Phys. Rev. D 89, 054014 (2014).

[62] B. Back et al., Phys. Rev. C 72, 031901(R) (2005).

[63] W. Busza, J. Phys. G 35, 044040 (2008).

[64] B. Abelev et al. (ALICE Collaboration), Phys. Rev. Lett. 110, 032301 (2013).

[65] J. Adam et al. (ALICE Collaboration), Phys. Rev. C 91, 064905 (2015).

[66] G. Aad et al.(ATLAS Collaboration), arXiv:1508.00848. 